\title{
Management of Preterm Infants with Respiratory Distress Syndrome Using Non-Invasive High Frequency Ventilation versus Nasal Continuous Positive Airway Pressure \\ Baha Elhadi Masoud Ettrmal*, Hisham Sami Abd Alhamid, Hosam Fathi Elsaadany \\ Pediatrics Department, Faculty of Medicine, Zagazig University, Egypt \\ *Corresponding author: Baha E. Ettrmal, Mobile: (+2):010281440 97, Email: bahabon2006@ gmail.com
}

\begin{abstract}
Background: Preterm infants have a risk of the respiratory distress syndrome (RDS). Helpful effects of high-frequency ventilators have been shown within management of RDS as well as the use of these ventilators as the initial mode of support or as a rescue treatment once failure of standard mechanical ventilation.

Objective: This study aimed to compare between the nasal high-frequency oscillatory ventilation (nHFOV) and nasal continuous positive airway pressure (NCPAP) in the treatment of preterm infants with RDS in.

Patients and methods: A study included 62 neonates with respiratory distress syndrome and was conducted at neonatal intensive care unit, Pediatrics Department, Zagazig University Hospital. Cases were divided into; group (A) was managed with non-invasive high frequency nasal ventilation and group (B) was managed with nasal continuous positive airway pressure. All cases were subjected to full medical history, clinical assessment, laboratory investigations and Silverman Score for respiratory distress grading.

Results: There was statistically non-significant difference between the studied groups regarding occurrence of nasal injury, oxygen supply after study and need for use of surfactant. There was statistically non-significant difference between the studied groups regarding $\mathrm{x}$ ray findings before or by the end of the study. There was statistically significant relation between gestational age groups and need for surfactant in NHFV group.

Conclusion: Noninvasive high frequency ventilation (NHFV) represent a better primary non-invasive mode than NCPAP in treatment of preterm infants with RDS.
\end{abstract}

Keywords: CPAP, Neonates, NHFV, RDS.

\section{INTRODUCTION}

Preterm birth is the leading reason behind newborn death worldwide. Preterm infants have a risk of the respiratory distress syndrome (RDS), which is one among the foremost common morbidities in preterm infants. The introduction of endotracheal ventilation has improved the survival rate among preterm infants however it is related to an increased risk of complications such as bronchopulmonary dysplasia (1).

In the past few decades, nasal ventilation has been used to manage and improve respiratory complications in patients with respiratory distress syndrome (RDS) ${ }^{(2)}$. Nasal continuous positive airway pressure (NCPAP) is the utilization of positive pressure to the airways of spontaneously breathing newborn during the respiratory cycle ${ }^{(3)}$.

The helpful effects of high-frequency ventilators have been shown within management of RDS as well as the use of these ventilators as the initial mode of support or as a rescue treatment once failure of standard mechanical ventilation ${ }^{(4)}$.

Sufficient recruitment of lung volume has the major part of protecting and preserving lung architecture in addition potentiating surfactant therapy ${ }^{(5)}$.

Nasal high-frequency oscillatory ventilation (nHFOV) is effective and superior to nasal intermittent positive pressure ventilation regarding lung carbon dioxide elimination during a model using newborn manikins. The nHFOV is a non-invasive ventilation mode that applies an oscillating pressure wave type to the airways using a nasal interface "nasal prong or face mask", This mode has been shown to facilitate carbon dioxide expiration, however very little is understood regarding its use in neonates ${ }^{(6)}$.

The current study aimed to compare between the nHFOV and NCPAP in the treatment of RDS in preterm infants regarding efficacy, safety, reducing the need for intubation and avoiding the complication of mechanical ventilation.

\section{PATIENTS AND METHODS}

This comparative non blind randomized clinical trial included 62 neonates with respiratory distress syndrome.

This study was conducted at neonatal intensive care unit, Pediatrics Department Zagazig University Hospital.

\section{Inclusion criteria:}

Preterm infant with a gestational age between 30 to 36 weeks and 6 days. Preterm infants with a good weight for the gestational age. Preterm infants with spontaneous breathing.

Preterm infants with respiratory distress syndrome, including all grades "Mild, moderate, severe" with signs and symptoms such as grunting, intercostals, subcostal retraction and cyanosis. Preterm infants with respiratory distress syndrome suggestive by chest $\mathrm{X}$-rays. 


\section{Exclusion criteria:}

Preterm infant with congenital abnormalities at birth, preterm infants who needed intubation for resuscitation. Preterm infants which developed one or more of these complications of RDS as atelectasis, pneumothorax, intra-cranial hemorrhage and disseminated intravascular coagulation "DIC".

\section{Ethical approval:}

The study was approved by the Ethical Committee of Zagazig Faculty of Medicine. An informed consent was obtained from the parents of all patients in this research. Every parent received an explanation for the purpose of the study. All given data were used for the current medical research only. This work has been carried out in accordance with The Code of Ethics of the World Medical Association (Declaration of Helsinki) for studies involving humans.

\section{Clinical assessment:}

All cases were subjected to full medical history from their parents, clinical and laboratory examination including: APGAR score, Silverman Score for respiratory distress grading. Chest X-ray and head ultrasound was performed. Cases were divided into two groups randomly as the follow: Group (A) was managed with non-invasive high frequency nasal ventilation, we used two types of ventilators to generate nasal high frequency ventilation, SLE 5000 infant ventilator (UK) and Drager babylog 800 plus (Germany). Initial setting: mean airway pressure: $6 \mathrm{~cm}$ $\mathrm{H}_{2} \mathrm{O}$, range (6-8) frequency of $10 \mathrm{~Hz}$, range (10-12), Amplitude or Delta pressure $12 \mathrm{~cm} \mathrm{H}_{2} \mathrm{O}$, range (10-20). The inspiratory time was adjusted to $50 \%(1: 1)$. $\mathrm{FiO}_{2}$ was adjusted to target oxygen saturation $\left(\mathrm{SpO}_{2}\right)$ above $90 \%$ by pulse oximeter. Baby connected to the ventilators by nasal connection: mini flow, silicon nasal prong and bonnet by MEDIN (Germany).

Group (B) was managed with nasal continuous positive airway pressure, with initial setting, mean airway pressure $6 \mathrm{~cm} \mathrm{H}_{2} \mathrm{O}$ range (6-8). Baby was connected to CPAP by nasal connection Lion medic infant circuit (Egypt). Surfactant was given to preterm with moderate and severe respiratory distress syndrome through endotracheal tube using proper size catheter, we used Alveofact (Germany).

To minimize abdominal distension, an orogastric tube was placed in the stomach and gas was going to be periodically aspirated during the study period in each group. Short binasal prongs was used in each group, and they changed periodically to reduce the chance of nasal injury.

\section{Weaning criteria:}

No or minimal sign of respiratory distress, positive end-expiratory pressure $<6 \mathrm{~cm} \mathrm{H}_{2} \mathrm{O}$ and Fio2 $<30 \%$.

\section{Evaluation of outcomes:}

Total time of use NHFV and time of use NCPAP and time needed for oxygenation for each case were recorded. Detection of complications that appear during noninvasive ventilation (pulmonary air leak syndrome, IVH, nasal injury) were detected.

\section{Statistical analysis}

Data were analyzed using the software SPSS (Statistical Package for the Social Sciences) version 20. Categorical variables were described as frequency and percentage and were compared using Chi square test. Kolmogorov-Smirnov (distribution-type) and Levene (homogeneity of variances) tests were used. Quantitative were presented as mean \pm standard deviation (SD) and were compared by Mann Whitney test (for not normally distributed data) and independent sample $\mathrm{t}$ test for normally distributed data. Paired sample $\mathrm{t}$ test and Wilcoxon signed tank tests were used to compare paired quantitative data. McNemar test was used to compare paired categorical data at two points of time within the same group. $\mathrm{P}$ value $<0.05$ was considered significant.

\section{RESULTS}

The present study revealed statistically nonsignificant difference between the studied groups regarding Silverman score before the study. On the other hand, there is statistically significant difference between the studied groups regarding Silverman score by the end of the study that lower in NHFW group. In each group, there is significant decrease in Silverman score over time (Table 1).

Table (1): Comparison between NHFV and NCPAP regarding Silverman score before intervention and Silverman score after study

\begin{tabular}{|l|c|c|c|}
\hline \multirow{3}{*}{ Silverman score } & \multicolumn{2}{|c|}{ Groups } & Test \\
\cline { 2 - 3 } & NHFV group & NCPAP group & \multirow{2}{*}{ P } \\
\cline { 2 - 4 } & $\mathrm{N}=31(\%)$ & $\mathrm{N}=31(\%)$ & 0.49 \\
\hline Before $($ Mean \pm SD) & $6.84 \pm 0.86$ & $7.0 \pm 0.97$ & $0.013^{*}$ \\
\hline After $($ Mean \pm SD) & $2.0 \pm 0.41$ & $3 \pm 0.68$ & \\
\hline P & $<0.001^{* *}$ & $<0.001^{* *}$ & \\
\hline
\end{tabular}

NHFV: Non-invasive high frequency nasal ventilation, NCPAP: Nasal continuous positive airway pressure, SD: standard deviation, *: Statistically significant, **: Statistically highly significant.

There was statistically significant difference between the studied groups regarding duration of treatment (significantly lower in NHFV group) (Table 2). 
Table (2): Comparison between NHFV and NCPAP regarding Duration of treatment (hours)

\begin{tabular}{|l|c|c|c|}
\hline \multirow{2}{*}{ Parameter } & \multicolumn{2}{|c|}{ Groups } & Test \\
\cline { 2 - 3 } & NHFV group & NCPAP group & \multirow{2}{*}{ p } \\
\cline { 2 - 3 } & $\mathrm{N}=31(\%)$ & $\mathrm{N}=31(\%)$ & \multirow{2}{*}{$\begin{array}{c} \\
\text { Duration (hours) } \\
\text { Mean } \pm \text { SD }\end{array}$} \\
\hline
\end{tabular}

NHFV: Non-invasive high frequency nasal ventilation, NCPAP: Nasal continuous positive airway pressure, SD: standard deviation, **: Statistically highly significant

There was statistically non-significant difference between the studied groups regarding occurrence of nasal injury, oxygen supply after study and need for use of surfactant (Table 3).

Table (3): Comparison between NHFV and NCPAP regarding clinical outcomes

\begin{tabular}{|c|c|c|c|}
\hline \multirow[t]{3}{*}{ Nasal injury } & \multicolumn{2}{|c|}{ Groups } & Test \\
\hline & NHFV group & NCPAP group & \multirow[t]{2}{*}{$\mathbf{p}$} \\
\hline & $\mathrm{N}=31(\%)$ & $\mathrm{N}=31(\%)$ & \\
\hline $\begin{array}{l}\text { Yes } \\
\text { No }\end{array}$ & $\begin{array}{c}4(12.9) \\
27(87.1)\end{array}$ & $\begin{array}{l}10(32.3) \\
21(67.7)\end{array}$ & 0.068 \\
\hline $\begin{array}{l}\text { Free } \mathrm{O}_{2} \\
\text { Nasal } \mathrm{O}_{2} \\
\mathrm{MV}\end{array}$ & $\begin{array}{c}0(0) \\
24(77.4) \\
7(22.6) \\
\end{array}$ & $\begin{array}{c}1(3.2) \\
18(58.1) \\
12(38.7) \\
\end{array}$ & 0.17 \\
\hline $\begin{array}{l}\text { Surfactant } \\
\text { Given } \\
\text { Not given }\end{array}$ & $\begin{array}{l}15(48.4) \\
16(51.6)\end{array}$ & $\begin{array}{l}14(45.2) \\
17(54.8)\end{array}$ & 0.799 \\
\hline
\end{tabular}

NHFV: Non-invasive high frequency nasal ventilation, NCPAP: Nasal continuous positive airway pressure, MV: mechanical ventilation

There was statistically non-significant difference between the studied groups regarding X-ray findings before or by the end of the study (Table 4).

Table (4): Comparison between NHFV and NCPAP regarding X-ray findings before and after

\begin{tabular}{|l|c|c|c|}
\hline \multirow{2}{*}{ CXR } & \multicolumn{2}{|c|}{ Groups } & \multirow{2}{*}{ Test } \\
\cline { 2 - 3 } & NHFV group & NCPAP group & \multirow{2}{*}{} \\
\cline { 2 - 3 } & $\mathrm{N}=31(\%)$ & $\mathrm{N}=31(\%)$ & \\
\hline Before & & $10(32.3)$ & \\
RD I & $7(22.6)$ & $14(45.2)$ & \\
RD II & $9(29.0)$ & $7(22.6)$ & \\
RD III & $15(48.4)$ & $19(61.3)$ & \\
\hline After & & $3(9.7)$ & 0.664 \\
Normal & $22(71)$ & $9(29)$ & \\
Air bronchogram & $3(9.7)$ & $6(19.4)$ & \\
Pneumonia & &
\end{tabular}

NHFV: Non-invasive high frequency nasal ventilation, NCPAP: Nasal continuous positive airway pressure

There was statistically significant relation between gestational age groups and need for surfactant in NHFV group (all very early preterm need surfactant) (Table 5).

Table (5): Relation between gestational age and need for surfactant among the studied groups

\begin{tabular}{|l|c|c|c|c|c|c|}
\hline \multirow{3}{*}{ Parameter } & \multicolumn{3}{|c|}{ NHFV group } & \multicolumn{3}{|c|}{ NCPAP group } \\
\cline { 2 - 7 } & Very preterm & Moderate preterm & Late preterm & Very preterm & Moderate preterm & Late preterm \\
\cline { 2 - 7 } & $\mathrm{N}=7(\%)$ & $\mathrm{N}=22(\%)$ & $\mathrm{N}=2(\%)$ & $\mathrm{N}=12(\%)$ & $\mathrm{N}=10(\%)$ & $\mathrm{N}=9(\%)$ \\
\hline Surfactant & & & & & & \\
Given & $7(100)$ & $7(31.8)$ & $1(50)$ & $6(50)$ & $5(50)$ & $3(33.3)$ \\
Not given & $0(0)$ & $15(68.2)$ & $1(50)$ & $6(50)$ & $5(50)$ & $6(66.7)$ \\
\hline P & & $0.007 *$ & & & 0.699 & \\
\hline
\end{tabular}

*: Statistically significant 


\section{DISCUSSION}

Neonatal RDS is a common respiratory disease, which occurs in neonates due to lack of the surfactant, which is secreted by type II pneumocyte, which helps the inflation of the alveoli during inspiration and prevent the atelectasis and collapse of the alveoli during expiration ${ }^{(7)}$. One of the most ordinarily used noninvasive strategies is nasal continuous positive airway pressure (NCPAP), which is comparatively easy and effective therapy in the early management of RDS in newborn ${ }^{(8)}$. Yet, some newborn with NCPAP therapeutic technique develops respiratory failure and need mechanical ventilator support ${ }^{(9)}$.

The current study was conducted on 62 preterm neonates with respiratory distress syndrome, who were divided into two equal groups: Group (A) received noninvasive high frequency nasal ventilation and group (B) received nasal continuous positive airway pressure. We aimed to compare between both groups in treatment of preterm neonates with RDS.

In our study, there was statistically significant difference in duration of treatment between the studied groups, which was significantly lower in NHFV group. This was in agreement with Shadbolt (10) who conducted a meta-analysis included 7 studies, which concluded that NIHFOV is more effective than NCPAP for reducing the duration of non-invasive ventilation (NIV).

In our study there was statistically nonsignificant difference between the studied groups regarding oxygen supply after treatment. This was in accordance with Malakian et al. ${ }^{(11)}$ who concluded that NHFOV did not decrease the need for mechanical ventilation compared with NCPAP overall in the first 72 hours of life; the rates of the primary outcome did not differ significantly between the NHFOV (6.5\%) and NCPAP $(14.1 \%)$ groups $(P=0.13)$. This was also in agreement with Zhu et al. ${ }^{(12)}$ who conducted a study in 18 tertiary neonatal intensive care units in China. A total of 302 preterm infants born at a gestational age (GA) of 26 weeks +0/7D -33 weeks +6/7D weeks with a diagnosis of RDS. They concluded that NHFOV was not superior to NCPAP with regard to the primary outcome when applied as the primary respiratory support for RDS in infants between $26+0 / 7$ and $33+6 / 7$ weeks of GA. This was in disagreement with Li et $\boldsymbol{a l}$. (13) who involved 463 patients. The meta-analysis estimated a lower risk of intubation (relative risk $=0.50$, $95 \%$ confidence interval of 0.36 to 0.70 ); NHFOV significantly reduced risk for intubation compared with nCPAP/BP-CPAP.

Our study showed statistically non-significant difference between the studied groups regarding need for use of surfactant. This was in accordance with Cao et al. ${ }^{(14)}$ who studied 512 preterm infants (gestational age of 26 weeks 0 day to 33 weeks 6 days) with RDS who received NHFOV or NIPPV or NCPAP after birth were analyzed, which showed statistically nonsignificant difference between the three studied groups.
Our study showed statistically non-significant difference between the studied groups regarding occurrence of nasal injury. This was in agreement with Malakian et al. ${ }^{(11)}$ who showed insignificant difference between the studied groups regarding traumatization of nasal skin and mucosa $(\mathrm{P}=0.260)$.

In our study, we applied NIHFV via binasal silicone prongs, in different sizes, which is the most common interface, used in European neonatal intensive care units (NICUs), as a new non-invasive ventilation mode, clinicians are concerned about the safety of NHFOV. A survey done in five European countries showed that abdominal distension, and upper airway obstruction due to viscous secretions were the most common side effects during NHFOV ${ }^{(6)}$.

\section{CONCLUSION}

Noninvasive high frequency ventilation (NHFV) represents a better primary non-invasive mode than NCPAP in treatment of preterm infants with RDS. An additional large randomized trials are needed to evaluate the use of noninvasive high frequency ventilation among smaller preterm infants as well as to compare different devices and approaches for administering noninvasive high frequency ventilation.

\section{Financial support and sponsorship: Nil. Conflict of interest: Nil.}

\section{REFERENCES}

1. Roberts C, Owen L, Manley B et al. (2016): Nasal high-flow therapy for primary respiratory support in preterm infants. $\mathrm{N}$ Engl J Med., 375(12): 1142-1151.

2. Iranpour R, Armanian A, Abedi A et al. (2019): Nasal highfrequency oscillatory ventilation (nHFOV) versus nasal continuous positive airway pressure (NCPAP) as an initial therapy for respiratory distress syndrome (RDS) in preterm and near-term infants. BMJ Paediatrics Open, 3(1): 29-33.

3. Cambonie G, Milési C, Jaber $\mathbf{S}$ et al. (2008): Nasal continuous positive airway pressure decreases respiratory muscles overload in young infants with severe acute viral bronchiolitis. Intensive Care Medicine, 34(10): 1865-1872.

4. Ramanathan R (2008): Optimal ventilatory strategies and surfactant to protect the preterm lungs. Neonatology, 93(4): 302308 .

5. Mammel M, Courtney S (2016): High-frequency ventilation. Assisted Ventilation of the Neonate E-Book. An Evidence-Based Approach to Newborn Respiratory Care: Sixth Edition. Elsevier Inc., Pp.211-228. https://doi.org/10.1016/B978-0-323-39006-4.00022-3

6. De Luca D, Dell'Orto V (2016): Non-invasive high-frequency oscillatory ventilation in neonates: review of physiology, biology and clinical data. Archives of Disease in ChildhoodFetal and Neonatal Edition, 101(6): 565-570.

7. Pickerd N, Kotecha S (2009): Pathophysiology of respiratory distress syndrome. Paediatrics and Child Health, 19(4): 153-157.

8. Fleeman N, Mahon J, Bates $\mathrm{V}$ et al. (2016): The clinical effectiveness and cost-effectiveness of heated humidified highflow nasal cannula compared with usual care for preterm infants: systematic review and economic evaluation. Health Technology Assessment, 20(30): 1-67.

9. DiBlasi R (2011): Neonatal noninvasive ventilation techniques: do we really need to intubate?. Respiratory Care, 56(9): 12731297. 
10. Shadbolt J (2019): Noninvasive high-frequency oscillatory ventilation in preterm infants with respiratory distress syndrome. Posters from the Canadian Society of Respiratory, 19: 142-148.

11. Malakian A, Bashirnezhadkhabaz S, Aramesh $M$ et al. (2018): Noninvasive high-frequency oscillatory ventilation versus nasal continuous positive airway pressure in preterm infants with respiratory distress syndrome: A randomized controlled trial. The Journal of Maternal-Fetal \& Neonatal Medicine, 33: 1-151.

12. Zhu X, Shi Y, Shi L et al. (2018): Noninvasive high-frequency oscillatory ventilation versus nasal continuous positive airway pressure in preterm infants with respiratory distress syndrome: Study protocol for a multi-center prospective randomized controlled trial. Trials, 19: 319-327.

13. Li J, Li X, Huang X et al. (2019): Non-invasive high-frequency oscillatory ventilation as respiratory support in preterm infants: A meta-analysis of randomized controlled trials. Respiratory Research, 20(1): 58-63.

14. Cao H, Li H, Zhu $X$ et al. (2020): Three non-invasive ventilation strategies for preterm infants with respiratory distress syndrome: a propensity score analysis. Archives of Medical Science, 16(6): 1319-26. 